\title{
Effect of Annealing Process on Microstructure of SiC-Mg Mixture Powder
}

\author{
Jingkun Li ${ }^{1}$, Xueping Ren ${ }^{1 *}$, Yanling Zhang ${ }^{2,3,4}$ and Hongliang $\mathrm{Hou}^{2,3,4}$ \\ ${ }^{1}$ School of Materials Science and Engineering, University of Science and Technology Beijing, China \\ ${ }^{2}$ AVIC Manufacturing Technology Institute, China \\ ${ }^{3}$ Aeronautical Key Laboratory for Plastic Forming Technology, China \\ ${ }^{4}$ Beijing Key Laboratory of Digital Forming Technology and Equipment, China
}

*Corresponding author: Xueping Ren, School of Materials Science and Engineering, University of Science and Technology Beijing, China

\begin{abstract}
$\mathrm{SiC}$ particles with 8wt.\% magnesium alloy powders as additives were mixed followed by annealing at 280 and $400{ }^{\circ} \mathrm{C}$ for 1 and $4 \mathrm{~h}$. The particle size, phase condition, morphology and compositions distribution were analyzed using laser diffraction analyzer, X-ray diffraction (XRD), scanning electron microscopy (SEM). It was found that particles mixed homogeneously before annealing. Annealing processes have a positive effect on the decrease of magnesium alloy particle size. It has almost no effect on magnesium alloy particle size with increasing the annealing duration at $280{ }^{\circ} \mathrm{C}$. However, the diameter of magnesium particle decreased obviously with increasing the annealing duration at $400{ }^{\circ} \mathrm{C}$. Magnesium alloy particles annealed at $400^{\circ} \mathrm{C}$ is smaller than that of the $280^{\circ} \mathrm{C}$ ones under the same annealing duration. Si component was observed in powder annealed at both temperatures. Grain size of $\mathrm{SiC}$ was reduced and microstrain was improved with increase of annealing temperature and duration.
\end{abstract}

Keywords: Powder technology; particles; annealing process; microstructure

\section{Introduction}

Silicon carbide $(\mathrm{SiC})$ is widely used in structural and functional materials [1-4]. Metals are widely used as additives in sintering SiC ceramic [5]. During sintering process, metals play a bonding role among $\mathrm{SiC}$ particles, which promote formation of sintering necks. In this way, densification process of $\mathrm{SiC}$ particles is accelerated $[1,6]$. In order to obtain ideal microstructure, the $\mathrm{SiC}$ powder is often pre-treated, such as coating additives and refining particles $[7,8]$. However, traditional methods require special instrument or take a long time. As we all know, diffusion occurs during annealing. While, microstructure and properties will be changed. This work intends to report effect of annealing processes on SiC-Mg mixture powder with different temperature and duration. Morphology, microstructure and component evolutions were characterized by $\mathrm{X}$-ray diffraction and SEM. Finally, average grain size and micostrain of SiC were discussed.

\section{Materials and Method}

SiC powder (purity > 99\%) and 8wt.\% magnesium alloy powder (purity $>99 \%$ ) were used as the raw materials in the present study. The powder was mixed in a three-dimensional mixing machine for $2 \mathrm{~h}$. The mixture was then poured in a glass tube with argon atmosphere. The mass of powder is $15 \mathrm{~g}$ in each group. Annealing processes were conducted using a SRJX-8-13 heat treatment furnace. Annealing temperature was set as 280 and 400 ${ }^{\circ} \mathrm{C}$ with 1 or $4 \mathrm{~h}$ separately. The 280 and $400{ }^{\circ} \mathrm{C}$ are temperatures of stress relief annealing and full annealing separately, and 1 and 4 $\mathrm{h}$ are general designed according to annealing time of magnesium alloy. Powder annealed at $280^{\circ} \mathrm{C}$ for 1 hour was termed as Powder 1 and the 4 hour one was termed as Powder 2. Similarly, Powder annealed at $400{ }^{\circ} \mathrm{C}$ for 1 hour was termed as Powder 3 and the 4 hour one was termed as Powder 4. Mass of powder was measured using an electronic balance. The particle size distribution was 
characterized using a laser particles analysis. The phase condition was characterized using an XRD analysis with $\mathrm{Cu}$ target between $30^{\circ}$ and $80^{\circ}$ at a $0.02^{\circ} / \mathrm{sec}$ step at $35 \mathrm{kV} / 40 \mathrm{~mA}$. Morphology and component were characterized using a LE01450 SEM with an Energy Dispersive Spectrometer (EDS).

\section{Results and discussion}
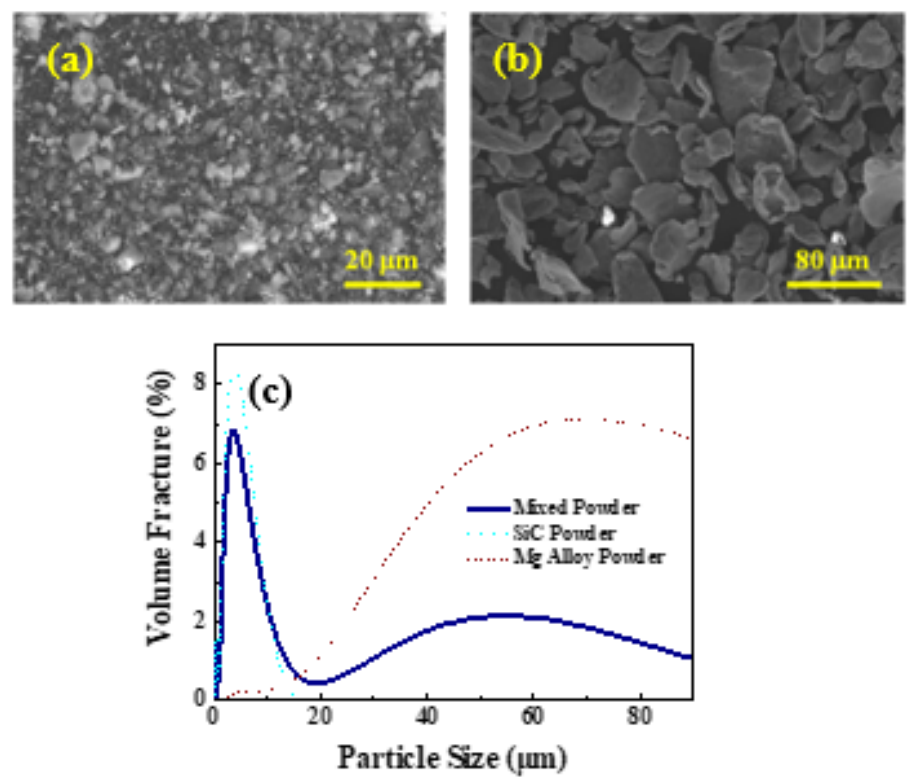

Figure 1: Morphology and particle size distribution before annealing A) Morphology of SiC particles, B) Morphology of magnesium alloy particles, C) Particle size distribution of $\mathrm{SiC}$, magnesium alloy and mixture.

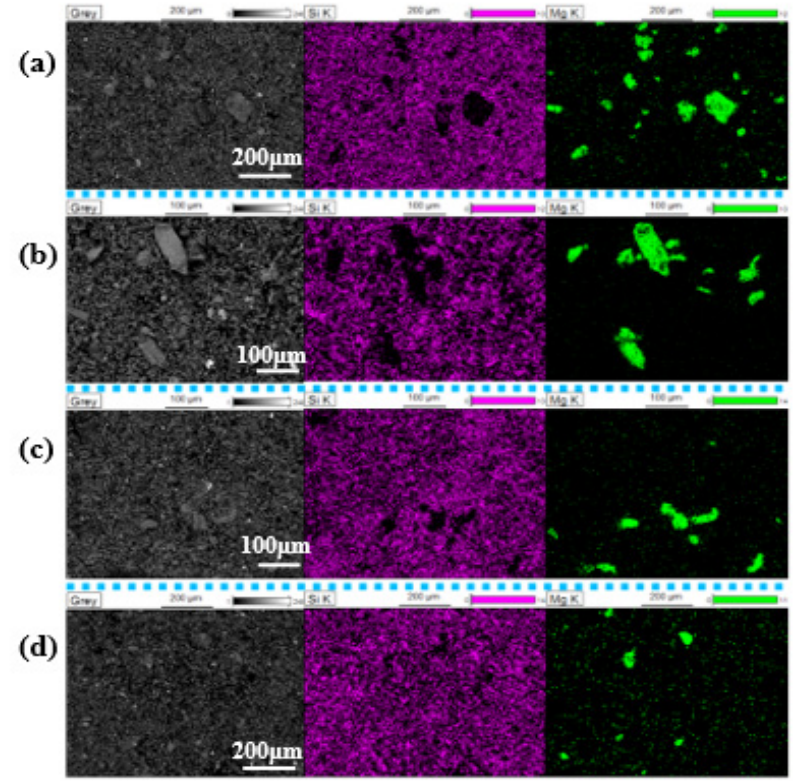

Figure 2: Morphology and Si, Mg element distribution of annealed powder A) Powder 1, B) Powder2, C) Powder 3, D) Powder 4. 
(a)

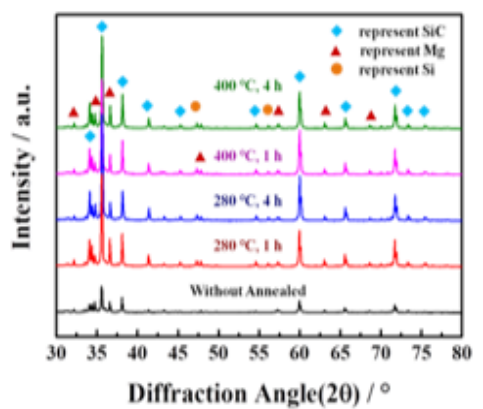

(b)

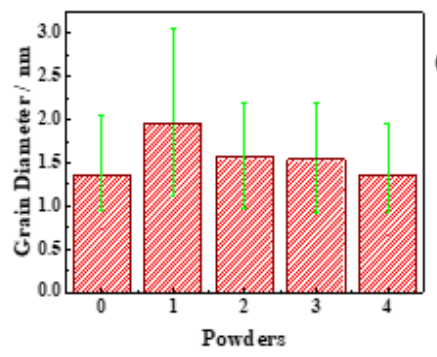

(c)

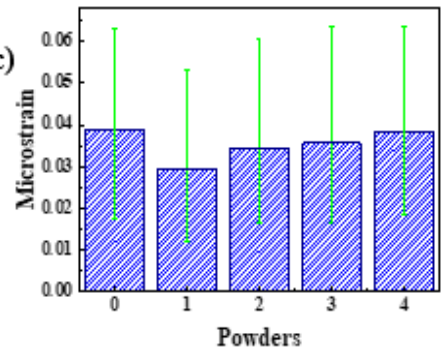

Figure 3: X-ray diffraction patterns of powder samples A) X-ray diffraction patterns, B) Calculated grain size, C) Calculated microstrain..

Table 1: Particle sizes of magnesium in Powder 1 to 4 .

\begin{tabular}{|c|c|c|c|c|}
\hline Powders & $\mathbf{1}$ & $\mathbf{2}$ & $\mathbf{3}$ & $\mathbf{4}$ \\
\hline $\mathrm{Mg}$ Particle Size/ $\mu \mathrm{m}$ & 59.33 & 58.35 & 50.49 & 43.9 \\
\hline
\end{tabular}

As Figure 1A \& 1B shows, $\mathrm{SiC}$ particles have an irregular polygon, whereas that of magnesium ones has a lamellar shape. After mixing, the distribution of particle size exhibits a bi-peak characteristic, which is similar with those of raw SiC and magnesium alloy powders, as shown in Figure 1C. The average particle size of $\mathrm{SiC}$ is $3.31 \mu \mathrm{m}$ and that of magnesium alloy is $86.4 \mu \mathrm{m}$. Since the powders are randomly sampled, it can be considered that $\mathrm{SiC}$ and magnesium alloy powders have been mixed homogeneously. Figure 2 shows morphology of annealed powder, where the distributions of Si (pink) and Mg (green) elements are inserted in the right side. As Figure 2 shows, it has almost no effect on the particle morphology of $\mathrm{SiC}$ with different annealing processes. However, both particle size and morphology of magnesium alloy are affected by the annealing process. Table 1 lists average particle size of magnesium alloy according to Figure 2. It has little effect on particle size of magnesium alloy with further increasing the annealing time at $280^{\circ} \mathrm{C}$. However, particle size of magnesium alloy can be obviously reduced after annealed at $400{ }^{\circ} \mathrm{C}$, and it can be further reduced with increasing the annealing duration. Figure 3 shows the phase analysis results using XRD with different annealing processes. Compared with that of raw powders, there is new diffraction peak of Si observed, indicating that $\mathrm{SiC}$ have decomposited partly during annealing. What's more, the orientation strength of annealed powders is enhanced, which indicates that microstructure have changed. In order to further character microstructure evolution of different annealing processes, grain size and microstrain of $\mathrm{SiC}$ are calculated using the XRD data, as shown in Figure 3B \& 3C, where " 0 " represents powder before annealing. For all the annealed powder, grain size of $\mathrm{SiC}$ increased and microstrain decreased after annealing, as presented in Figure 3B \& 3C. Gain size of SiC annealed at $400{ }^{\circ} \mathrm{C}$ is smaller than that of $280{ }^{\circ} \mathrm{C}$ with the same annealing time, and it decrease with prolongation of annealing time.

Previous studies shown that the (111) crystal plane of SiC matches well with the (0001) plane of Mg [9]. So, the improvement of annealing temperature and prolongation of annealing time provide opportunities for $\mathrm{SiC}$ and magnesium to complete full reflection. Meanwhile, $\mathrm{SiC}$ has an positive effect on refining magnesium grain, and the $\mathrm{C}$ element decomposed from $\mathrm{SiC}$ plays a role of carbonaceous inoculation treatment [10]. Furthermore, a little Al among the magnesium alloy powder can also promote interaction between $\mathrm{SiC}$ and $\mathrm{Mg}$ [11]. Microstrain presents the contract tendency with grain size evolution. In general, powder with smaller particle size, smaller grain size and higher microstrain is an ideal candidate for sintering [12]. So, a complex milling process is usually introduced $[13,14]$. However, in this letter, the above target has achieved by powder mixing followed by simple and efficient annealing. According to present study, the mixture powder annealed at $400{ }^{\circ} \mathrm{C}$ for $4 \mathrm{~h}$ is the ideal candidate for sintering.

\section{Conclusion}

a) Annealing have almost no effect on the particle morphology of $\mathrm{SiC}$ but it can reduce particle size of magnesium alloy. Particle size of magnesium alloy annealing at $400{ }^{\circ} \mathrm{C}$ is smaller than the 
$280^{\circ} \mathrm{C}$ ones with the same annealing duration. Prolongation of annealing time has little effect on particle size of magnesium at $280^{\circ} \mathrm{C}$. However, it has a positive effect on particle size reduction when annealed at $400{ }^{\circ} \mathrm{C}$.

b) The orientation strength of annealed powders is enhanced, which indicates that microstructure have changed during annealing. New diffraction peak of Si observed, indicating that $\mathrm{SiC}$ would have decomposited partly during annealing.

c) Grain size of $\mathrm{SiC}$ decreased and microstrain increased with the improvement of annealing temperature and prolongation of annealing duration at 280 and $400{ }^{\circ} \mathrm{C}$ for 1 and $4 \mathrm{~h}$. Powder annealed at $400{ }^{\circ} \mathrm{C}$ for $4 \mathrm{~h}$ with the smallest grain size and largest microstrain is the ideal candidate of sintering.

\section{References}

1. Wu R, Zhou K, Yue CY, Wei J, Pan Y (2015) Recent progress in synthesis, properties and potential applications of $\mathrm{SiC}$ nanomaterials, Prog Mater Sci 72: 1-60.

2. Chaika AN, Aristov VY, Molodtsova OV (2017) Graphene on cubic-SiC, Prog Mater Sci 89: 1-30.

3. Taubner T, Korobkin D, Urzhumov Y, Shvets G, Hillenbrand R (2006) Near-field microscopy through a SiC superlens, Science 313 (5793): 1595.

4. Vakifahmetoglu C, Zeydanli D, Colombo P (2016) Porous polymer derived ceramics, Mat Sci Eng R 106: 1-30.
5. Zhu M, Wang Y (2009) Pressureless sintering ZrB2-SiC ceramics at low temperatures, Mater Lett 63(23): 2035-2037.

6. Moberlychan WJ, Cao JJ, Jonghe LCD (1998) The roles of amorphous grain boundaries and the $\beta-\alpha$, transformation in toughening $\mathrm{SiC}$, Acta Mater 46(5): 1625-1635.

7. Shao J, Li M, Chang K, Huang Y, Ren D, et al. (2018) Fabrication and characterization of SPS sintered SiC-based ceramic from Y3Si2C2-coated SiC powders, J Eur Ceram Soc 38(15): 4833-4841.

8. He Q Wang A, Liu C, Wang W, Wang H (2018) Microstructures and mechanical properties of B4C-TiB2-SiC composites fabricated by ball milling and hot pressing, J Eur Ceram Soc 38(7): 2832-2840.

9. Luo A, (1996) Heterogeneous nucleation and grain refinement in cast Mg (AZ91)/SiCp, metal matrix composites, Can Metall Quart 35(4): 375383.

10. Qian M, Cao P (2005) Discussions on grain refinement of magnesium alloys by carbon inoculation, Scripta Mater 52(5): 415-419.

11. Easton MA, Schiffl A, Yao JY, Kaufmann H (2006) Grain refinement of Mg$\mathrm{Al}(-\mathrm{Mn})$ alloys by $\mathrm{SiC}$ additions, Scripta Mater 55(4): 379-382.

12. Yu H, Sun Y, Hu L, Zhou H, Wan Z (2016) Microstructural evolution of AZ61-10at. \%Ti composite powders during mechanical milling, Mater Design 104: 265-275.

13. Albaaji AJ, Castle EG, Reece MJ, Hall JP, Evans SL (2017) Effect of ballmilling time on mechanical and magnetic properties of carbon nanotube reinforced FeCo alloy composites, Mater Design 122: 296-306.

14. Suryanarayana C (2001) Mechanical alloying and milling, Prog Mater Sci 46(1-2): 1-184.

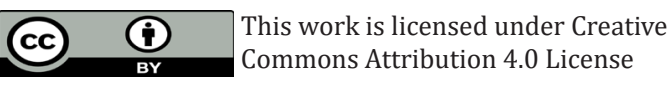

To Submit Your Article Click Here: Submit Article
DOI: $10.32474 / J O M M E .2019 .01 .000106$

Citation: Jingkun Li, Xueping Ren, Yanling Zhang, Hongliang Hou. Effect of Annealing Process on Microstructure of SiC-Mg Mixture Powder. J Mining \& Mech Eng 1(2)- 2019. JOMME.MS.ID.000106. DOI: 10.32474/JOMME.2019.01.000106.

- Unique DOI for all articles

\section{Journal Of Mining And Mechanical Engineering}

\section{Assets of Publishing with us}

- Global archiving of articles

- Immediate, unrestricted online access

- Rigorous Peer Review Process

- Authors Retain Copyrights 